\title{
DIFFERENTIAL CALCULUS: THE USE OF NEWTON'S METHODUS FLUXIONUM ET SERIERUM INFINITARUM IN AN EDUCATION CONTEXT \\ Volume 65,2015
}

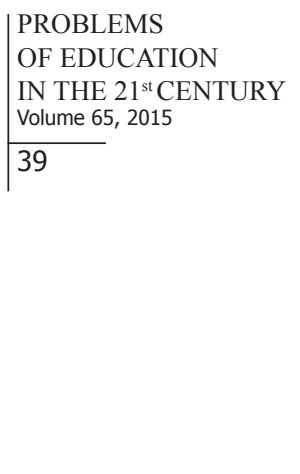

PROBLEMS

IN THE $21^{\text {st }}$ CENTURY

\author{
Paolo Bussotti \\ University of Udine, Italy \\ E-mail: paolobussotti66@gmail.com
}

\begin{abstract}
What is the possible use of history of mathematics for mathematics education? History of mathematics can play an important role in a didactical context, but a general theory of its use cannot be constructed. Rather a series of cases, in which the resort to history is useful to clarify mathematical concepts and procedures, can be shown. A significant example concerns differential calculus: Newton's Methodus fluxionum et serierum infinitarum is a possible access-key to differential calculus. For, many concepts introduced by Newton ought be useful for the pupils/students (last or last but one year at the high school and first year at the university) to reach a more intuitive, geometrical and problem-oriented approach to calculus. The motivation to consider history of mathematics as an important didactical support is that the pupils/students often learn mathematics in a too formal manner, without understanding the real reasons for the introduction of several mathematical concepts. The problem is that the potential of such support is not exploited. The educational proposal is hence to show a concrete case to highlight what the teaching of mathematics based on history means. The conclusion is that a general theory, as differential calculus, should be considered by the pupils/students as a necessity, deriving from a specification, improvement and extension of the techniques used to solve significant problems posed and developed in the course of history. In this manner, mathematics appears as a human activity comparable with other activities and not as a merely formal exercise.
\end{abstract}

Key words: mathematics education, history of mathematics, Newton, fluxions, tangents, maxima and minima, problem solving approach to mathematics education.

\section{Introduction}

The research here expounded concerns a broad debate: what is the role of history of mathematics for mathematics education? Numerous examples can be offered to approach this question: one of them concerns the problems of maxima, minima and tangents, which can be introduced, following the method conceived by the great French mathematician Pierre de Fermat (1601-1665) (Bussotti, 2014). Although Fermat did not develop the concept of derivative in a proper sense, the explanation how he faced the question maxima-minima-tangents can be instructive to offer an intuitive and problem-centred approach to the way in which infinitesimal quantities can be treated. A methodology based on problem solving and on the way in which along history of mathematics - some questions were addressed, might be more interesting for the students and more fruitful, from an educative standpoint, than a traditional series of lessons, in which the concepts of limit, derivative, integral and series are introduced by formal steps. A completely intuitive approach to mathematics is not fruitful: it is well known that intuition can induce serious mistakes and that a formalization is necessary for a correct mathematical reasoning. Nevertheless, in an initial educative phase, the resort to intuitive argumentations and to an approach based on specific problems can be useful for the pupils/students to catch the mathematical world in which they are operating. Beyond this, a more "concrete" education 
Paolo BUSSOTTI. Differential calculus: The use of Newton's methodus fluxionum et serierum infinitarum in an education context

PROBLEMS

OF EDUCATION

IN THE $21^{\text {st }}$ CENTURY

Volume 65,2015

might be more fascinating for the pupils/students, as they are immediately involved in a series of reasoning of which they can be an active part and not only passive listeners. Inside an education oriented towards this direction, history of mathematics can assume an important role. History of mathematics is not a universal key-access to mathematics education, but in many circumstances, it can be a useful and stimulating support, because of several reasons:

1) It presents to the students the way of reasoning of genial mathematicians, whose approach was different - in the methods and the language - from that proposed in the handbooks. This depends, first of all, on the fact that these mathematicians did not have a general theory of the problems they were trying to solve. They themselves were constructing such a theory. Therefore, many concepts and procedures were not yet codified. Moreover, they were writing to spread their ideas to other scientists and mathematicians, not to students. This means that the argumentation is often not plain. Thence, if the teacher/professor drives the students inside these argumentations and, afterwards, he/she develops a comparison with the formal modern approach, the result ought be a complete, clear and satisfying educational scenario;

2) The way in which great men tried to solve some mathematical problems as well as the difficulties and failures they had to address, is a quite significant social and moral lesson: no result are obtained without labour and work. Mathematics and science are not born codified. Many efforts have been necessary for a discipline to reach a satisfying form. History is the ideal field for the pupils/students to catch this feature, which connotes all human activities and which, hence, goes far beyond the specific content of mathematics.

In this context, history of mathematics is not the main subject. The history of mathematics is a quite specialized field of research and it is different from mathematics education. This means that an absolute precision from a historical point of view is not necessary while introducing history of mathematics in mathematics education. It is important that the concepts and procedures of the mathematicians are expounded with a certain precision - otherwise this approach is useless and even noxious -, but it is not needed to enter into many refined problems typical of history of mathematics and science. For example: when we refer to a method used by a certain author, it is not necessary to specify that other authors used that method, too, and it is not required to enter into questions as the priority of a certain discovery or procedure.

Actually, the precise explanation of the concepts and of the problems the mathematician was facing is fundamental rather than a series of refined historiographical questions, which, in an educative context, are inappropriate. In this paper, the guidelines here outlined are followed.

The core of the research concerns two mathematical problems developed by Newton (1642-1727) in his Methodus fluxionum et serierum infinitarum (Newton 1671, 1736, 1746):

1) Development into infinite series of expressions and equations;

2) Calculus of the fluxions, in particular referred to: a) maxima, minima, tangents; b) curvature of a flat line at a given point.

This work by Newton can be symbolically considered the birth certificate of differential and integral calculus. Differently from most of Newton's works, it is written also in an educative - or at least explicative - perspective. As to the two previous items, the development into infinite series is a decisive step towards Newton's solutions of several differential equations and towards the integration by series developed by him. In this research, such subjects are not dealt with, but the item 1) represents an introduction, which can be exploited in educative terms. The calculus of the fluxions is, in practice, the differential calculus. Thence the choice of item 2) needs no explanation.

The selection of a specific work is arbitrary, at least to a certain extent. However, Newton's Methodus fluxionum et serierum infinitarum offers, inside a sole book, a stimulating exposition of many of the problems which connote the differential and integral calculus. Obviously, other choices could be equally valid.

For each subject addressed in this research, some of Newton's results are expounded 
and, in a separate sub-section, presented as a box, a series of didactical considerations is added, which, hopefully, might be stimulating both for teachers/professors and pupils/students to reach an approach, where elements drawn from history of mathematics are added in a systematic manner.

Since mathematical analysis is such an important discipline, it is almost pervasive. In the Italian educative system (but this is true for most of the European educative systems), mathematical analysis is taught in the last or last but one year of the scientific and technical high school, when the pupils are 18-19 years old. After that, in the faculties of mathematics, physics and many branches of engineering there are two examinations: one concerning the functions of one real variable (here the same subjects taught at the high school are explained, though in a far more profound and formalized manner), the other one concerning the function of two real variables. In many other faculties - as informatics, chemistry, economy, biology, only to mention someone - there is a more or less difficult examination of mathematics, in which calculus gets a preponderant role.

The educative approach is rather classical. First of all, the concept of function is introduced, after that, the one of limit, with the operations with the limits and some theorems connected to their main features. The next step is the concept of derivative with a series of theorems and applications of the differential calculus (the problem of maxima, minima and tangents enters into these applications, as well as the explanation that velocity is the first derivative of space, in respect to time and acceleration, the first derivative of velocity). The integral calculus and - at the university - the study of the series (both numerical series and series of functions) follow.

As to the functions of two variables, the partial derivatives and the total differential are introduced as well as the concept of gradient with conspicuous elements of vector analysis. The problems of relative, absolute and constrained maxima and minima for functions of two variables follow. An important part of the programme concerns the exact differentials. With regard to the integral calculus, the double and triple integrals are explained as well as the linear and superficial integrals. The notions of rotor and divergence are faced and the solutions of several kind of differential equations are offered.

In the faculties of mathematics, conspicuous elements of set theory and concepts as Euclidean space, Hilbert space and normed space are introduced, notions which will be fundamental for the advanced studies addressed by the students in the following years.

Although, as it is easy to imagine, there are differences from school/university to school/ university, depending on the programs and on the educative convictions of the teachers/professors, the world of the mathematical analysis is presented, step by step as a unitary and wellfounded building. Certainly this is not the way in which this world was constructed during history. Thus: can history be of help to better clarify - also from an educative point of view - some concepts and procedures, so that the students can catch and appreciate the mathematical methods they are learning, as something vivid?

To answer this question a specific methodological approach is necessary. For, it is needed:

a) Adescriptive analysis of the way in which the mathematician, whose ideas are considered, structured his work;

b) The translation of his ideas into a language which can be easily understood by the pupils/students;

c) The organization of a series of educative steps to clarify that an approach to mathematics education based on history is often preferable to that characterising most of the handbooks.

During this research, such a methodological approach is followed. 
Paolo BUSSOTTI. Differential calculus: The use of Newton's methodus fluxionum et serierum infinitarum in an education context

\section{PROBLEMS \\ OF EDUCATION \\ IN THE $21^{\text {st }}$ CENTURY \\ Volume 65,2015 \\ 42 Newton's Infinite Series and Fluxions Calculus}

Newton dedicated several of his works or part of works to calculus. At least three contributions have to be mentioned:

1) Methodus fluxionum et serierum infinitarum, written around 1671, but published posthumously in 1736 in an English version, which is the translation of the original Latin one, published ten years later, in 1746 (see Newton 1671, 1736, 1746);

2) the first section of the first book of the Philosophiae Naturalis Principia Mathematica (Newton 1687, 1713, 1726, 1739-1742).

3) De quadratura curvarum, published in 1704 on the basis of manuscripts dating back to the beginning of the 90' (see Newton 1704, 1746).

Newton slightly changed his approach to calculus in the course of the years, but the basic ideas remained unmodified. In this context, the focus is on the Methodus fluxionum et serierum infinitarum.

\section{Newton's Method to Develop Some Mathematical Expressions in Infinite Series}

The Methodus fluxionum is a significant text in our context: this is the work in which Newton introduced his concepts of fluxion and fluent. As told, from a symbolical point of view, it could be considered the birth of the differential calculus. However - differently from other quite specialized and brachylogical works as the Principia or the Enumeratio linearum tertii ordinis - the Methodus was also conceived as an educational work, in which the reader is introduced step by step inside the faced subjects. Newton explicitly wrote:

I thought it not amiss, for the sake of young Students in this Science, to compose the following Treatise, in which I have endeavoured to enlarge the Boundaries of Analytics, and to improve the Doctrine of Curve-lines (Newton 1671, 1736, p. 1).

Ideally the Methodus can be divided into three parts.

First part: Newton develops the technique of the infinite series.

Second part: he introduces the concept of fluxion and: a) given a fluxional equation with the fluxions of two quantities, he teaches how to obtain the ratio between the two fluxions. Here he also refers to what previously analysed as to the infinite series; b) solves the problems of the maxima and minima of a given curve and of the tangent to a curve from a point of it; c) solves the problem concerning the curvature of a line.

Third part: Newton shows how to calculate the areas of curve flat regions and how to measure the length of a curve. Here, both the infinite series and the fluxions play a fundamental role. This is what nowadays we call integral calculus.

As to the development of expressions and equations in series, the initial idea by Newton is the attempt to offer a general method to solve an entire series of problems by approximation, if it is not possible to solve them offering the exact solution, which can be caused both by a very mathematical impossibility or by a not yet sufficient knowledge of such problems. His approach is completely different from that used in modern mathematical education. For, nowadays the development of a function in a series (for example series of Taylor or of McLaurin) is introduced only once the concept of function, limit and derivative have been explained. Furthermore, for a series to represent the development of a function, it is necessary that such a series is uniformly convergent. Thence, a conspicuous theoretical preparation is necessary. Newton did not have exactly the concept of function and derivative, he only outlined to the problem of the series' convergences and adopted, in this case, a pragmatic procedure, which, nevertheless, worked for the numerous cases he presented and which can be considered a general method. An explanation of Newton's methodologies is useful: 
1) are possible; for the pupils to understand how many different approaches to mathematics

2) To realize that mathematics originates from specific problems and is not born and does not function as the well established and formed theory, which is presented in the school/university.

This might stimulate the creativity of the pupils/students.

The basic idea behind the method of the infinite series is, given an expression, in which a certain quantity is considered a variable, to write the whole expression as a power series of that quantity. In a quite clear, didactical manner, which is unusual in Newton, he explained how to divide the fraction

The scheme is this:

$$
\frac{a^{2}}{b+x}
$$

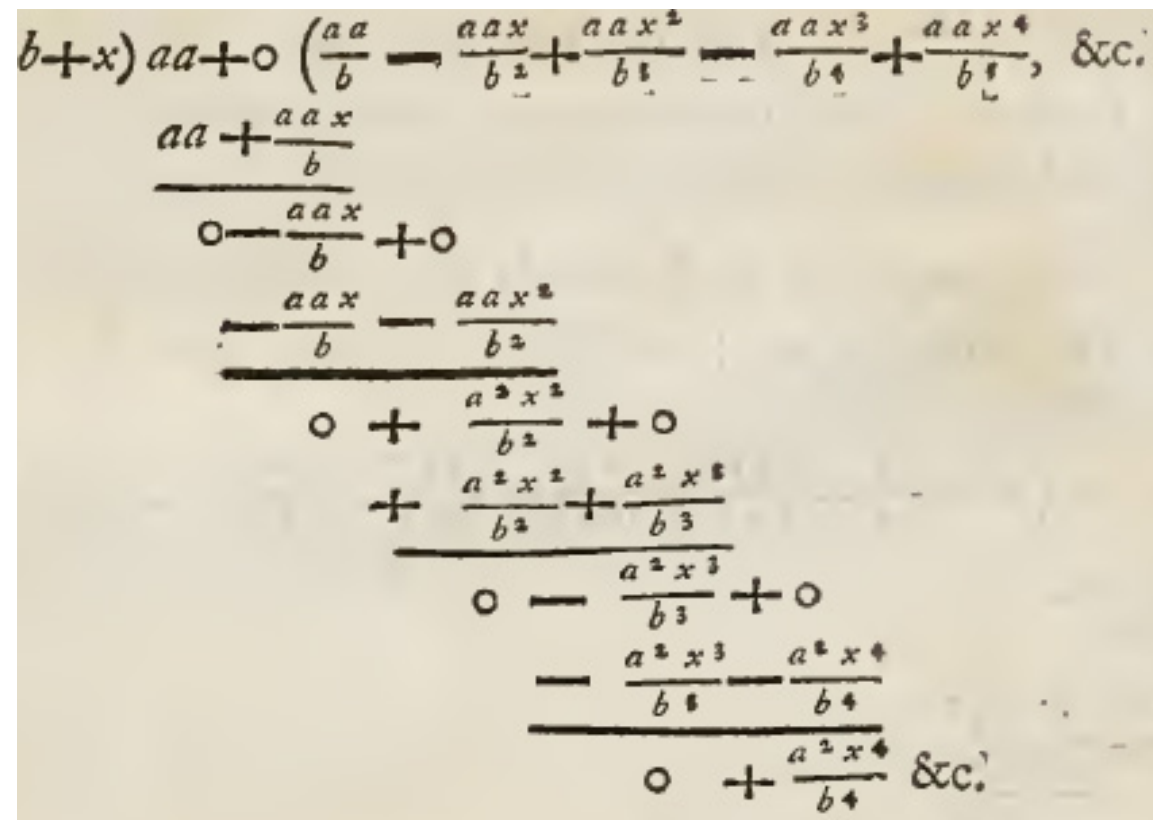

Figure 1: Newton's scheme for the division of $a^{2}$ by $b+x$. From Newton 1736, p. 3.

The explanation is the following one: Newton divides $a^{2}$ by $b$ and obtains $\frac{a^{2}}{b}$. This is the first digit of the quotient. After that, he multiplies $\frac{a^{2}}{b}$ by $\quad b+x$, obtaining $a^{2}+\frac{a^{2} x}{b}$. He proceeds as in an ordinary division, subtracting $a^{2}+\frac{a^{2} x}{b}$ from $a^{2}$. The rest is $-\frac{a^{2} x}{b}$, which, divided by $b$, gives $-\frac{a^{2} x}{b^{2}}$. This is the second digit of the quotient.

The procedure continues now in the same manner indefinitely. Therefore the development of 
Paolo BUSSOTTI. Differential calculus: The use of Newton's methodus fluxionum et serierum infinitarum in an education context

PROBLEMS

OF EDUCATION

IN THE $21^{\text {st }}$ CENTURY

Volume 65,2015

$\frac{a^{2}}{b+x}$, according the powers of $x$ is

$$
\frac{a^{2}}{b+x}=\frac{a^{2}}{b}-\frac{a^{2} x}{b^{2}}+\frac{a^{2} x^{2}}{b^{3}}-\frac{a^{2} x^{3}}{b^{4}}+\ldots
$$

By following the same procedure, the algebraic fractions containing irrational expressions can

be developed, too. For example, Newton shows that the development of $\frac{2 x^{\frac{1}{2}}-x^{\frac{3}{2}}}{\frac{1}{2}}$ is

$$
1+x^{\overline{2}}-3 x
$$

$$
\frac{2 x^{\frac{1}{2}}-x^{\frac{3}{2}}}{1+x^{\frac{1}{2}}-3 x}=2 x^{\frac{1}{2}}-2 x+7 x^{\frac{3}{2}}-13 x^{2}+34 x^{\frac{5}{2}}+\ldots
$$

In the development of this expression, the exponents of the $x$ are fractions. In the years in which Newton wrote the Methodus fluxionum, he had already discovered the development of the binomial $(1+x)^{\theta}$, where $\theta$ represents any integral (also negative) or rational number. Therefore, the rational powers could be developed into series of powers with an integral exponent. Notwithstanding, Newton felt the need to show a plurality of cases and methods - as well as possible abbreviated procedures - in which a direct development was possible, without resorting to the binomial.

The following example offered by Newton is quite instructive and useful from an educational point of view, because it shows the relations between the calculation with numbers and the calculations with letters in a manner in which the rules of algebra might hide. Sometimes, these relations are not clear either in the high schools. The problem is to calculate the square root of a literal expression. Newton reasons in this way: let us suppose we have to develop the expression $\left(a^{2}+x^{2}\right)^{\frac{1}{2}}$.

Newton presents the following scheme. 


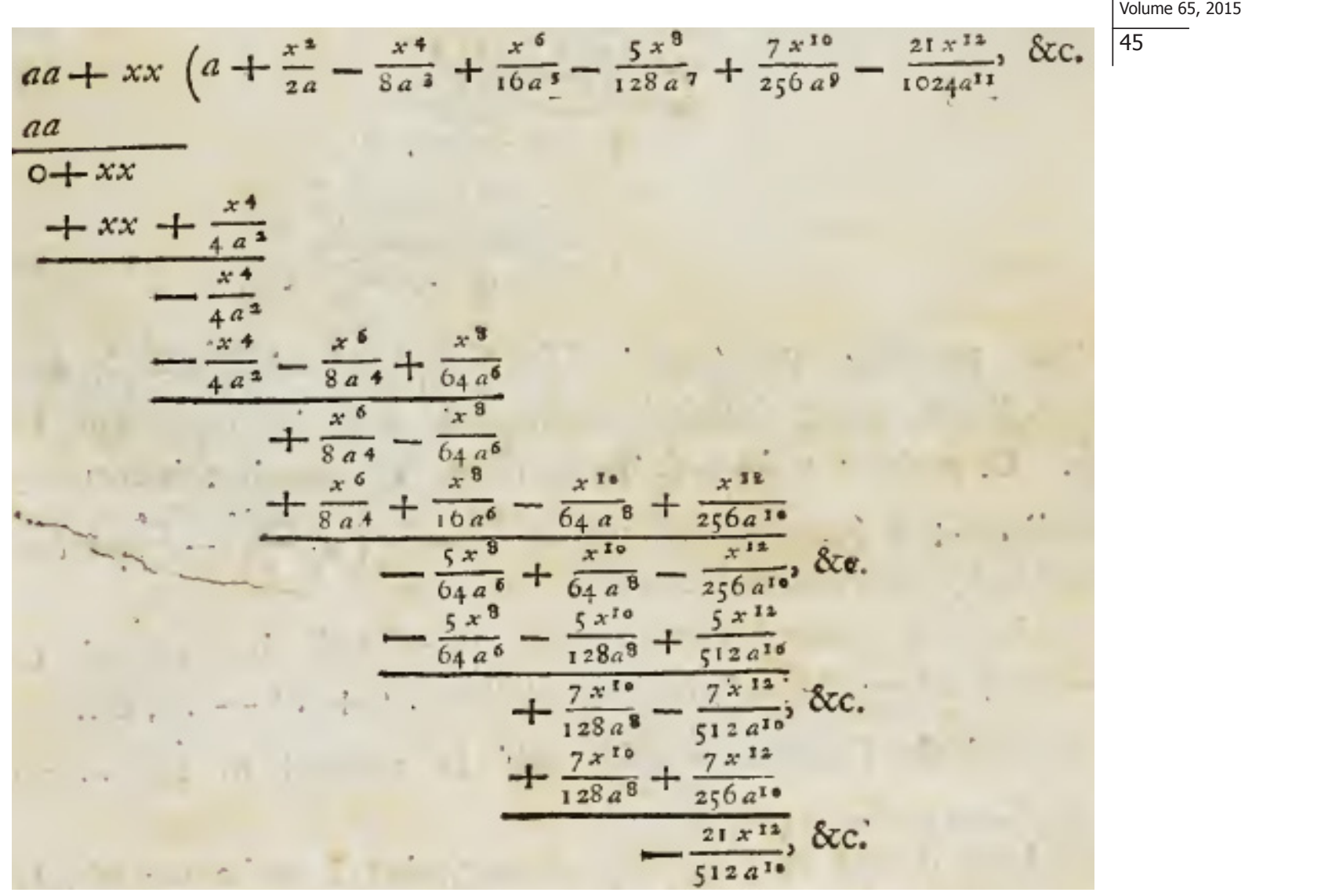

Figure 2: Newton's scheme for the calculation of $\left(a^{2}+x^{2}\right)^{\frac{1}{2}}$. From Newton 1736, p. 4.

The scheme is constructed like this: He considers the square root of $a^{2}$, that is $a$, which is the first element of the development. Written $a^{2}$ and carried out the subtraction $\left(a^{2}+x^{2}\right)-a^{2}$, we get the reminder $x^{2}$. Now, as is the case of the extraction of the square root of an integer, Newton multiplies the first element of the result, $a$, by 2 and divides the reminder $x^{2}$ by $2 a$, thus obtaining the second element of the result $\frac{x^{2}}{2 a}$. He executes the multiplication

$$
\left(2 a+\frac{x^{2}}{2 a}\right)\left(\frac{x^{2}}{2 a}\right)=x^{2}+\frac{x^{4}}{4 a^{2}}, \text { subtracts this value from } x^{2} \text { and obtains }-\frac{x^{4}}{4 a^{2}} .
$$

Now the procedure can be iterated: $-\frac{x^{4}}{4 a^{2}}: 2 a=-\frac{x^{4}}{8 a^{3}}$ is the third element of the result.

It is, thus, necessary to develop the multiplication

$$
\left(2 a+\frac{x^{2}}{a}-\frac{x^{4}}{8 a^{3}}\right)\left(-\frac{x^{4}}{8 a^{3}}\right)=-\frac{x^{4}}{4 a^{2}}-\frac{x^{6}}{8 a^{4}}+\frac{x^{8}}{64 a^{6}}
$$


PROBLEMS

OF EDUCATION

IN THE $21^{\text {st }}$ CENTURY

Volume 65,2015 as Newton claims,

We have to subtract this value from $-\frac{x^{4}}{4 a^{2}}$ and the procedure can be iterated, obtaining,
wton claims,

$$
\left(a^{2}+x^{2}\right)^{\frac{1}{2}}=a+\frac{x^{2}}{2 a}-\frac{x^{4}}{8 a^{3}}+\frac{x^{6}}{16 a^{5}}-\ldots
$$

Didactical considerations: it could be useful to show that this direct procedure supplies the same result as those offered by the development of the binomial. This could also be the occasion to discuss with the pupils/students with regard to general and more indirect methods - as, in this case, the binomial - and direct and more specific procedures as the one expounded. This might be fruitful from an educational point of view. A further interesting question is that the successive sums of an odd number of addends offer an approximation by defect of $\left(a^{2}+x^{2}\right)^{\frac{1}{2}}$, whereas the successive sums of an even number of addends offer an approximation by excess. This is not difficult to be seen and proved.

For example: $a<\left(a^{2}+x^{2}\right)^{\frac{1}{2}} ; a+\frac{x^{2}}{2 a}>\left(a^{2}+x^{2}\right)^{\frac{1}{2}} ; a+\frac{x^{2}}{2 a}-\frac{x^{4}}{8 a^{3}}<\left(a^{2}+x^{2}\right)^{\frac{1}{2}}, \ldots$;

The teacher/professor could tackle this problem: why does this procedure offer a progressively improving approximation of $\left(a^{2}+x^{2}\right)^{\frac{1}{2}}$, but once the value is smaller and once is greater than the original expression, while when we calculate the square root of an integer by the standard method, we get progressively better approximations, but the obtained values are always less that required root? This is not a difficult question, but I think the answer could be interesting in an educational perspective.

After having discussed the problems connected to the development in an infinite series of the algebraic expressions, Newton analyses a set of questions related to the solution of the equations by approximation, but in our context, what is interesting is the procedure by which he separates the variables in an algebraic expression, written nowadays, in the form $f\left(x^{m}, y^{n}\right)=0$ , where $m$ and $n$ are integers. However, as Newton points out, his method is far more general and works for all those expressions which, by the replacement of variables or other algebraic devices, can be reduced to the previous one. Newton deals with this problem inside his theory of equations, because he has the aim of expressing one variable of an equation as a series of powers of the other variable. The solution proposed by Newton is something genial, and it can be quite instructive for the pupils/students to understand what to invent, to create in mathematics means. The general method can be better explained starting from Newton's example: he considers the equation

$$
y^{6}-5 y x^{5}+\frac{x^{3} y^{4}}{a}-7 a^{2} x^{2} y^{2}+6 a^{3} y^{3}+b^{2} x^{4}=0
$$

The aim is to develop $y$ in a series of powers of $x$. To each equation a rectangle, composed of squares, can be associated. In this rectangle, the progressive powers of $x$ are represented in the first column from the left and the progressive powers of $y$ in the first line from below. The place of order $(m, n)$ (where the element of place 1,1, is that below on the left) represents the product $x^{m} y^{n}$. 
The scheme is like this:

$$
\begin{array}{cccccc}
x^{4} & x^{4} y & x^{4} y^{2} & x^{4} y^{3} & x^{4} y^{4} & . \\
x^{3} & x^{3} y & x^{3} y^{2} & x^{3} y^{3} & x^{3} y^{4} & . \\
x^{2} & x^{2} y & x^{2} y^{2} & x^{2} y^{3} & x^{2} y^{4} & . \\
x & x y & x y^{2} & x y^{3} & x y^{4} & . \\
1 & y & y^{2} & y^{3} & y^{4} & .
\end{array} .
$$

This scheme can be simplified - from a graphical point of view - by the following device: Newton associates such diagram to our equation:

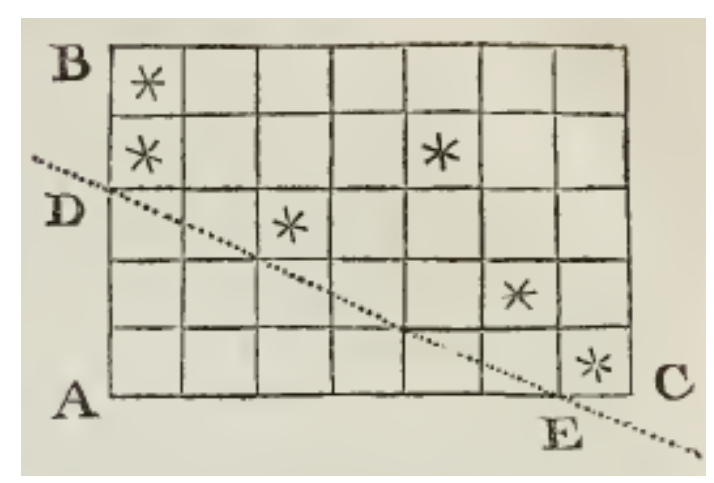

Figure 3: The scheme associated by Newton to equation 1). From Newton 1736, p. 10

whose meaning is (let us start from the first line below): the lowest exponent of $x^{0} y^{n}$ is 6 (this is the meaning of the six empty places in the first line); the lowest exponent of $x^{1} y^{n}$, is $n=5$; the lowest exponent of $x^{2} y^{n}$, is $n=2$; the lowest exponent of $x^{n} y^{0}$ is 3 (but there is also the exponent 4 , this is meaning of the higher asterisk in the first column); the lowest exponent of $x^{3} y^{n}$, is $n=4$. Therefore, all the elements of our equation are posed inside this scheme. Now Newton imagines that a ruler, starting from the first column on the left, passes through to the lowest exponents of $x^{n} y^{0}$ (in this case $x^{3}$ ). Supposing the ruler aligned along the exterior margin of the first column, let us think it rotates around the point $D$ as a centre until it touches the first element in which the variable $y$ exists. In this case, the ruler will touch the elements $(1,7)$ and $(3,3)$, which are associated to $x^{2} y^{2}$ and $y^{6}$. Newton selects hence the following equation from the original one:

$$
y^{6}-7 a^{2} x^{2} y^{2}+6 a^{3} y^{3}=0
$$

This equation has the evident solutions $y= \pm \sqrt{a x} ; y= \pm \sqrt{2 a x}$. It is legitimate to consider, as Newton does, one of these values as the first term of the development of $y$ in function of $x$. Two observations:

1) No one of the four values exactly solves the original equation. This means that the procedure continues by replacing, in the original equation, the value $\sqrt{a x}+p$ to $y$ (obviously 
Paolo BUSSOTTI. Differential calculus: The use of Newton's methodus fluxionum et serierum infinitarum in an education context

PROBLEMS

OF EDUCATION

IN THE $21^{\text {st }}$ CENTURY

Volume 65, 2015

each one of the four values $y= \pm \sqrt{a x} ; y= \pm \sqrt{2 a x}$ could be chosen). It is also possible to replace the radical by a new unknown $v$. Now a further equation is obtained to which the same procedure can be applied, reaching so progressively better approximations of $y$ written as a function of the powers of $x$.

2) Some problems could arise, which are solved by Newton through ingenuous algebraic devices. I only mention the most important one: it is possible that the first derived equation as, in our case $y^{6}-7 a^{2} x^{2} y^{2}+6 a^{3} y^{3}=0$ does not get evident solutions or cannot be solved algebraically. In this case, too, Newton is able to conceive some replacements of variables, which allow him to extract a solvable equation from an unsolvable one.

To make the procedure completely clear, I present a further example by Newton, to which a scheme is associated, as the scheme in figure 3, the first derived equation and the development of $y$.

Table 1. Development into series of a further equation.

\begin{tabular}{|c|c|c|c|}
\hline \multicolumn{4}{|c|}{ Equation: $y^{3}+a x y+a^{2} y-x^{3}-2 a^{3}=0$} \\
\hline$*$ & ${ }^{*}$ & & \\
\hline & ${ }^{*}$ & & \\
\hline & Derived equation: $y^{3}+a^{2} y-2 a^{3}=0$, solution $y=a$ \\
\hline \multicolumn{3}{|c|}{ Development of $y$ in function of $x$} \\
\\
$y$ \\
$y=a-\frac{x}{4}+\frac{x^{2}}{64 a}+\frac{131 x^{3}}{512 a^{2}}+\frac{509 x^{4}}{1638 a^{3}}+\ldots$ \\
\end{tabular}

Didactical considerations: in this case, too, a series of interesting didactical questions can be posed:

1) If we use different solutions of the first derived equations, we get different developments of $y$ : what are the relations between such developments?

2) How good is the approximation proposed by Newton?

3) How sound are his methods?

A discussion with the pupils/students about these problems could be quite fruitful, because this is an approach which is relatively unusual and which can be stimulating.

Inside his approach to calculus, the development in the series is fundamental for Newton because he exploited it in several circumstances, for example, he used what nowadays we call the integration by series and used the infinite series to solve differential equations. This is the reason why I have introduced this part of Newton's procedure. 


\section{The Concepts of Fluxion and Fluent}

The way in which Newton introduces the concepts of fluxion and fluent is coherent with two presuppositions of his: 1) the origin of these concepts derives from the necessity to use instantaneous quantities in physics; 2) the reference point of calculus are the infinite series.

Thus, the two problems to solve are the one the inverse of the other and precisely: i) given the space, to find the velocity, for any time; ii) given the velocity to find the space, for any time.

So that, Newton prosecutes: if the space is represented by the equation $y=x^{2}$, the ve-

locity is given by $2 x x$, where $x$ is the fluxion, in respect to time, of the fluent quantity $x$. Newton clarifies that the fluxions can be calculated in respect to time, but also in respect to any other variable, so that his analysis is not limited to a physical situation, but is more general. Newton offers a canon to calculate the equation of the fluxions, given the equation of the fluents. He starts from an equation of the fluents, in which only integral exponents appear. The canon is this:

a) to consider each variable separately;

b) to dispose the equation of the fluents according to the decreasing exponents of each variable, for example $x$;

c) let us consider the highest exponent $m$ of the variable $x$ : i) it is necessary to associate

an integer $n$ to such exponent; ii) to multiply each occurrences $x^{i}$ by $\frac{x}{x}$ and by the terms of

a decreasing arithmetical progression in this way: if we associate the number $n$ to $m$, we will multiply the occurrences of the variables with exponent $m$ by $n$; the occurrences with exponent $m-1$, by $n-h$ ( $h$ is, thus, the difference of the progression) and, in general, the occurrences $m-k$ by $n-k h$. Usually, it is posed $m=n$, and $h=1$, but this is not necessary.

d) to repeat this operation for each variable;

e) to sum the partial results.

The global result will provide the equation of the fluxions.

Let us analyse one of the examples proposed by Newton: let the equation of the fluents

$$
2 y^{3}+x^{2} y-2 c y z+3 y z^{2}-z^{3}=0
$$

be given. Let us calculate the fluxions for each variable in the order $x, y, z$. 
PROBLEMS

OF EDUCATION

IN THE $21^{\text {st }}$ CENTURY

Volume 65, 2015

Table 2. Relation Variable-Fluent-Fluxion.

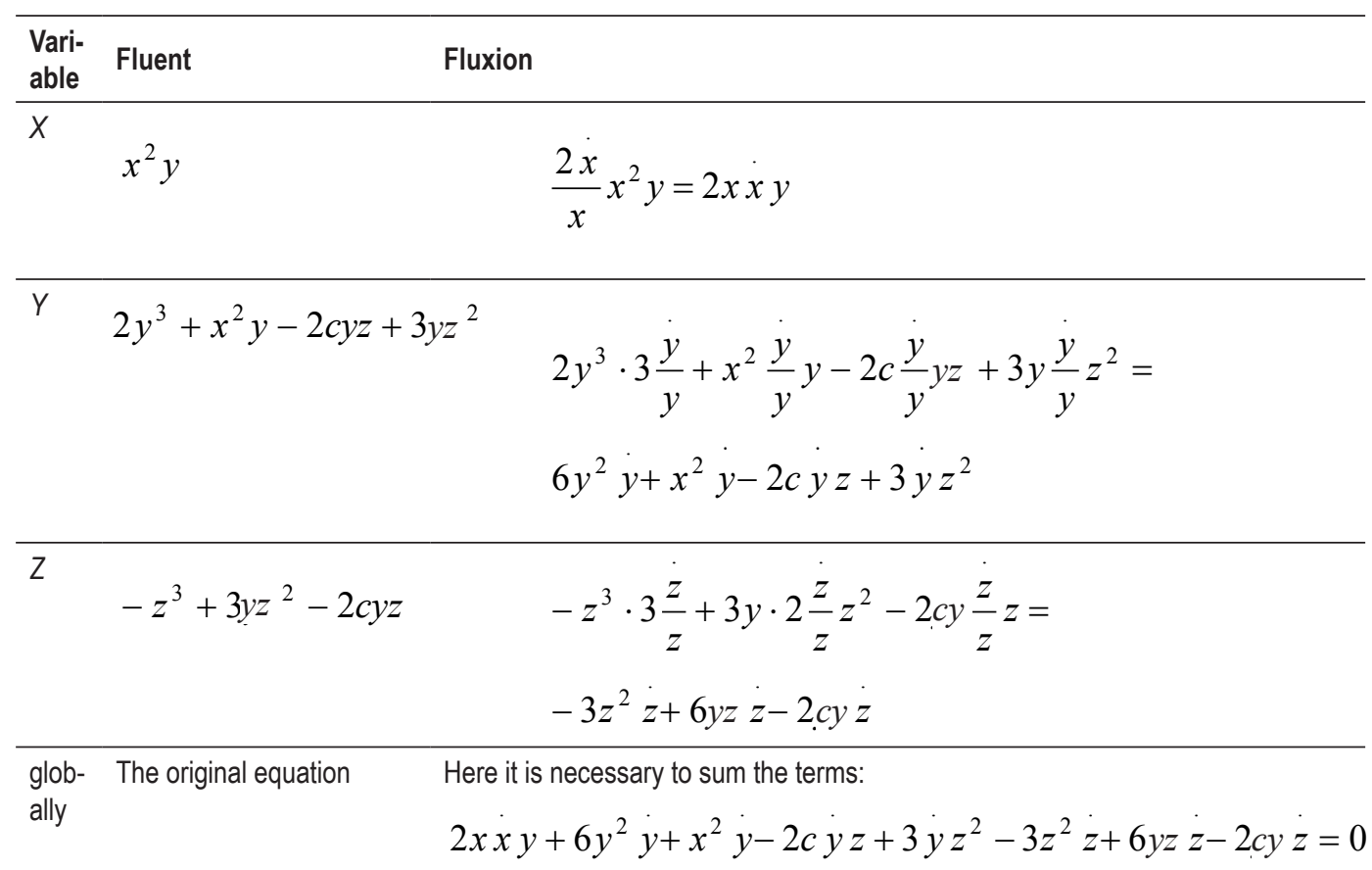

In this case the arithmetical progression by which to multiply the variables has been constructed assuming $m=n, h=1$. However, Newton offers another possibility (and several others could be conceived). His idea is to abbreviate as much as possible the equation of the fluxions. This is obtained, in our example, considering the variable $y$ : Newton associated the number 0 to $y^{1}$. Thence, to the expression containing $y^{2}$, the number 1 will be associated; to the expressions containing $y^{3}$, the number 2 , and to the expressions containing $y^{0}$, the number -1 . Thus, the scheme for $y$ will be like this:

Table 3. The fluxions calculated in respect to $y$.

\begin{tabular}{|l|l|l|}
$2 y^{3}+x^{2} y-2 c y z+3 y z^{2}-z^{3} y^{0}=0$ & $\begin{array}{l}2 y^{3} \cdot 2 \frac{\dot{y}}{y}+x^{2} y \cdot 0 \frac{y}{y}-2 c y \cdot 0 \frac{y}{y} z+3 y \cdot 0 \frac{\dot{y}}{y} z^{2}-z^{3} \cdot(-1) y^{0} \frac{\dot{y}}{y}= \\
4 y^{2} \dot{y}+z^{3} \frac{y}{y}\end{array}$
\end{tabular}

So that, the equation of the fluxions is:

$$
2 x \dot{x} y+4 y^{2} \dot{y}+\frac{y}{y} z^{3}-3 z^{2} z+6 y z \dot{z}-2 c y \dot{z}=0 .
$$

Newton also explains how to derive the fluxions' equation when irrational radical quantities exist in the fluent equation. Rather than an examination of this problem, a picture of how Newton justifies his procedures will be offered. A series of didactical and conceptual considerations will follow. 
Since all the variables are imagined as functions of $t$, Newton considers an indefinitely small quantity of time (quantitatem indefinite parvam, is the Latin expression, Newton 1746, p. 59), indicating it by $o$. The symbol $o$ can be interpreted as time-momentum, though Newton does not define it. The Momenta of the fluent quantities are given by the product of the fluxion by the time-momentum. If we consider, for example $x$, Newton defines its momentum as $x o$.

He justifies now his rule to get the fluxions equation by means of a paradigmatic example, through the following reasoning:

Let the equation

$$
x^{3}-a x^{2}+a x y-y^{3}=0
$$

be given. Newton considers the equation to which, for any variable, the variable itself, plus its momentum is replaced, thence the equation:

$$
(x+\dot{x} o)^{3}-a(x+\dot{x} o)^{2}+a(x+\dot{x} o)(y+\dot{y} o)-(y+\dot{y} o)^{3}=0
$$

If one develops all the factors and takes into account that $x^{3}-a x^{2}+a x z-y^{3}=0$, one gets:

$3 \dot{x} o x^{2}+3 \dot{x}^{2} o^{2} x+x^{3} o^{3}-2 a x o x-a x^{2} o^{2}+a x o y+a \dot{y} o x+a \dot{x} y o^{2}-3 y o y^{2}-3 \dot{y}^{2} o^{2} y-y_{y} o^{3}=0$

Now Newton divides this equation by $o$ and gets a further equation, in which some terms are affected by $o$, while other terms are not. As $o$ is an indefinitely little quantity, the terms affected by $o$ can be deleted, thus reaching the equation

$$
3 \dot{x} x^{2}-2 a \dot{x} x+a x y+a \dot{y} x-3 y y^{2}=0
$$

This results concludes the fundamental introductory part of Newton's fluxions-calculus. The observations which can be developed in an education perspective are numerous and interesting.

\section{Didactical considerations:}

A) The concept of function. The first question concerns the concept of function: the acquisition of this concept was slow, enough. The mathematician Peter Lejeune Dirichlet (1805-1859) in 1837 offered the modern definition of function. However, the work by Newton was fundamental towards the clarification, or at least, towards the use, of the concept of function. For, from the way in which Newton calculated his fluxions, it is evident that he considers the variables he uses $x, y, z$, .. mutually independent. However, all of them are variables depending on time, this means that a functional link with time exists. Furthermore, as we have seen, Newton specifies that the base-variable (nowadays we call it independent variable) is not necessarily the time. Newton does not develop a theory of the functional dependence. In particular, he does not deduce the relation between two variables exterminating the time because this dependence on time always remains a generic dependence. Newton does not write explicitly $x=f(t), y=g(t)$, as necessary in order to determine a functional link between $x$ and $y$. In brief: there is no theory of the functions in Newton. On the other hand, exactly the idea to consider all the variables as functions of time introduces to some interesting considerations. In particular, this seems to go towards the idea of a parametrization of the curves. Obviously this is only an outline because a necessary condition for a parametrization of a curve, for example in $\mathfrak{R}^{2}$ is to write the curve $\gamma(x, y)$ like this:

$$
\gamma(x, y)=\left\{\begin{array}{l}
x=f(t) \\
y=g(t)
\end{array}\right.
$$


Paolo BUSSOTTI. Differential calculus: The use of Newton's methodus fluxionum et serierum infinitarum in an education context

PROBLEMS

OF EDUCATION

IN THE $21^{\text {st }}$ CENTURY

Volume 65, 2015

While, as already pointed out, such specification does not exist in Newton. Nonetheless, it seems to me that the outlined-basic idea of a parametrization exists. Hence, in an educative perspective, these questions can be posed: i) what are the similarities and the differences between the relations among variables used by Newton and the modern concept of function? ii) What about the basicvariable time used by Newton and the modern parametrizations? iii) Could an educative approach, in which these concepts are introduced à la Newton, that is without a particular theoretical preparation, be useful and fruitful? The first two questions might develop a discussion between pupils/students and teachers/professors, whereas the last one is an argument of reflection for the teachers/professors.

B) Fluxions, fluents, momenta. Let us translate - where possible - the Newtonian language into the Leibnizian (and modern) one:

\begin{tabular}{|ll|}
\hline Newtonian Language & Leibnizian language \\
\hline $\begin{array}{l}\text { Indefinitely little quantity of time } \\
o\end{array}$ & $\begin{array}{l}\text { Time differential (though in Leibniz time does not get a privileged } \\
\text { status) } \\
d t\end{array}$ \\
\hline $\begin{array}{l}\text { Fluxion of the quantity } x \\
x\end{array}$ & $\frac{d x}{d t}$ \\
\hline $\begin{array}{l}\text { Momentum of the flowing quantity } x \\
x o\end{array}$ & $\begin{array}{l}\text { Differential of } x \\
d x\end{array}$ \\
\hline
\end{tabular}

An important difference is that $x$ indicates the fluxion of $x$ in respect to $t$, whereas Leibniz conceived $d x$

$\overline{d t}$ as a relation between two independent quantities.

Here, from an educative point of view, there are two relevant questions: i) Newton does not speak explicitly of limit, which is the fundamental concept of modern analysis. However, how close is the concept of "indefinitely little quantity of time" to that of limit? In this sense a further Newtonian procedure - introduced later on by the English scientist -, the "method of the first and last reasons" (see, for example, Newton 1704, 1746), could be introduced to develop an interesting discussion on the concept of limit; ii) what are the relations between the modern concept of derivative and the Newtonian fluxions? A first answer is: since neither the concepts of function and of limit yet existed, the Newtonian fluxion is not a derivative, but it is neither exactly the ratio between the two differentials of Leibniz. In Leibniz the two variables are completely independent, while, in Newton there is a dependence from $t$, though one of the main points of Newton's procedures is to consider $x, y, z, \ldots$ mutually independent. A quite interesting educative discussion is to compare the three concepts: Newtonian fluxions, Leibniz's differentials, modern derivatives. This would allow pupils/students to profoundly enter into the logic of mathematics.

C) Calculus of the fluxions: given an equation in which the exponents of the variables are integral numbers, Newton claims that the fluxions-equation in respect to a variable $x$ is o tained by: a) or-

dering the powers of that variable; b) dividing by $\frac{x}{x}$; c) multiplying the powers

of $x$ by any descending arithmetical progression, as seen above. The items a) and b) are plain, but c) is not. Let us explain this reconsidering the example of the equation

$$
2 y^{3}+x^{2} y-2 c y z+3 y z^{2}-z^{3}=0
$$




$$
2 x \dot{x} y+6 y^{2} \dot{y}+x^{2} \dot{y}-2 c \dot{y} z+3 \dot{y} z^{2}-3 z^{2} \dot{z}+6 y z \dot{z}-2 c y \dot{z}=0
$$

because, using a Newtonian language, our progression begins with the highest exponent of the variable and its difference is 1 . But how is obtained the canon, according to which each arithmetical progression can be used to gain the fluxions-equation. In our case: why are equation 2) and

$$
2 x \dot{x} y+4 y^{2} \dot{y}+\frac{\dot{y}}{y} z^{3}-3 z^{2} \dot{z}+6 y z \dot{z}-2 c y \dot{z}=0
$$

equivalent? Let us consider equation 1) and multiply it by $y^{-1}$, thus obtaining

$$
2 y^{2}+x^{2}-2 c z+3 z^{2}-\frac{z^{3}}{y}=0
$$

Let us now calculate the fluxions-equation by our usual procedure. We will have:

$$
4 \dot{y} y+2 \dot{x} x-2 c \dot{z}+6 z z-\frac{3 z z^{2}}{y}+\frac{z^{3} \dot{y}}{y^{2}}=0
$$

Finally, multiplying by $y$, one gets Newton's equation 3 ). Therefore, supposing $y \neq 0$, the equations 2) and 3) are equivalent. This clearly explains what means that any arithmetical progression can be used to obtain the fluxions-equation: different progressions are applied multiplying the original equation by $x^{m} y^{n}$ (in our case $m=0 ; n=-1$ ) and operating as shown.

With regard to this whole order of problems, an observation seems to me necessary: if we consider the expression $2 y^{3}+x^{2} y-2 c y z+3 y z^{2}-z^{3}$ as $f(x, y, z)=2 y^{3}+x^{2} y-2 c y z+3 y z^{2}-z^{3}$, its total derivative - in our language - is exactly

$2 x \dot{x} y+6 y^{2} y+x^{2} \dot{y}-2 c \dot{y} z+3 \dot{y} z^{2}-3 z^{2} z+6 y z \dot{z}-2 c y z$, which is, obviously, different from $2 x \dot{x} y+4 y^{2} \dot{y}+\frac{y}{y} z^{3}-3 z^{2} z+6 y z z-2 c y z$. Nonetheless - as pointed out -, the equations 2 ) and 3 ) are equivalent supposing $y \neq 0$. From this observation an interesting conceptual and didactical question arises: Newton passes from an equation of the fluents to a series of equivalent equations of the fluxions, while, given a function, the total derivative is only one. What is the relation between Newton's approach based on equations and an approach based on functions? The relations are strong and the two approaches, if applied to the same problems, have to provide the same solutions: but by means of which steps? This question is important in an educative perspective and can be useful to clarify the difference between an equation and a function, which, for the pupils/students is not always plain and to give for granted.

A brief historical observation: the mathematician Johannes Hudde (1628-1704) had developed the method to multiply a polynomial - ordered according to the descending powers of a variable - by any arithmetical decreasing progression, associating the highest number to the highest degree of the variable. This allowed Hudde to solve a series of problems concerning the decompositions of the polynomials and their maxima and minima (see Suzuki 2005). Newton mentions Hudde, who, anyway, did not reach the concept of fluxion or differential. 
PROBLEMS

OF EDUCATION

IN THE $21^{\text {st }}$ CENTURY

Volume 65, 2015

D) Rules of fluxions'calculus and independence of the variables. Newton - differently from Leibniz (Leibniz, 1684) with his differentials - does not offer a complete series of rules for the calculus of the fluxions. Nevertheless, these rules can be easily deduced from the cases he analyses. For example, if we consider the already seen equation $x^{3}-a x^{2}+a x y-y^{3}=0$ and the associated equation of the fluxions $3 \dot{x} x^{2}-2 a x x+a x y+a y x-3 y y^{2}=0$, it is clear that the fluxion of axy is $a x y+a y x$, which, in modern terms, is the rule for the derivative of the product of two functions. Furthermore, the fact that Newton considers all the variables $x, y, z, \ldots$ reciprocally independent and depending on time, allows him to treat the fluxions expressed by equations in which a variable alone exists - for example $x$ - in the same manner in which several variables appear. The Newtonian calculus is uniform, whatever the number of variable is: if we consider an equation of the fluents, for example, $E(x, y, z)=0$, the operations carried out by Newton to get the equation of the fluxions, can be interpreted like this in modern terms:

$$
\dot{E}(x, y, z)=\frac{\partial E}{\partial x} \frac{\partial x}{\partial t}+\frac{\partial E}{\partial y} \frac{\partial y}{\partial t}+\frac{\partial E}{\partial z} \frac{\partial z}{\partial t}=0
$$

In an educative perspective: 1) this is an immediate access-key both towards the rule of derivation of a composite function, towards the concept of partial derivative and of total derivative; 2) questions: a) how is this interpretation faithful to Newton's thought? b) what is the relations between these Newtonian concepts and the modern ones? c) Is it possible to present mathematical analysis in a unitary perspective starting from Newton's notions? I think this order of problems is interesting from an educational point of view.

\section{Maxima, Minima, Tangents}

Maxima and minima. Newton is clear in the presentation of his method to find the maxima and the minima of a certain expression posed equal to 0 : necessary condition for the expression to get a maximum or a minimum is that the fluxions of all the variables has the value 0 . This depends on the observation that a positive fluxion indicates that the values of the given expression are increasing, while a negative fluxion that they are decreasing. Thence, it is necessary that - to use a modern language - in a neighbourhood of the possible maximum or minimum the fluxion maintains the value 0 .

Newton offers immediately an example: he considers the already seen equation

$$
x^{3}-a x^{2}+a x y-y^{3}=0
$$

and its associated fluxions-equation

$$
3 \dot{x} x^{2}-2 a \dot{x} x+a \dot{x} y+a \dot{y} x-3 y y^{2}=0
$$

Let us consider the variable $x$. Then for the expression written in equation 3 ) to get a maximum or a minimum, it is necessary that $x=0$, thence arriving at

$$
3 y^{2}=a x
$$

Once obtained in this manner the value for $x$ or $y$, it is possible to replace it in 3 ), to get the value of the other variable, to replace it once again in 5) and to achieve the values for both the two variables.

Obviously: it is also possible to proceed considering the system, which derives from the 
annihilation of both $\dot{x}$ and $\dot{y}$. In this case the result is

$$
\left\{\begin{array}{l}
3 y^{2}-a x=0 \\
3 x^{2}-2 a x+a y=0
\end{array}\right.
$$

Newton points out the superiority of his method in comparison to other methods for the determination of the maxima and minima because it can be uniformly extended to quantities involving roots of any degree, which is not possible with the other methods. Newton provides the example of the equation

$$
x^{2}-a y^{2}+\frac{b y^{3}}{a+y}-x^{2} \sqrt{a y+x^{2}}=0
$$

By means of not difficult considerations, which, however, Newton does not offer in general in his work, it is possible to determine if the obtained points are very maxima or minima for the proposed expression.

Didactical considerations: from an educative point of view, there is a consideration, which is coherent with what pointed out at the end of the previous section: if we consider the whole expression posed equal to 0 as $f(x, z, y, w, \ldots)$, we see that Newton's method is exactly the same as that used to find the necessary condition for a point to be a maximum or a minimum, that is the annihilation of the partial derivatives of the function with respect to all the variables. Thence, this is a confirmation that a conspicuous series of problems can be treated by the same method, following Newton's approach. The differential calculus for the function of a variable appears to be treatable in a similar manner as the differential calculus for the functions of more than one variable. A quite interesting question is: till which point can this uniform approach be extended? This a stimulating argument of discussion, especially for the teaching of differential calculus in the two beginning years at the university.

Let us now consider the method proposed by Newton to determine the tangent to a curve. He offered eight different versions, of which the first one will be analysed.

Tangents. The problem of the tangents is treated by Newton analogously as that of maxima and minima. The point of maximum or minimum is considered as that to which the increasing and the decreasing value of the curve converge and the tangent is considered as the line to which the secants converge. This is the basic idea. However, both the problem of maximaminima and tangents are treated by Newton without the explicit resort to something like a limit. Newton reasons in this manner: 
PROBLEMS

OF EDUCATION

IN THE $21^{\text {st }}$ CENTURY

Volume 65,2015

56

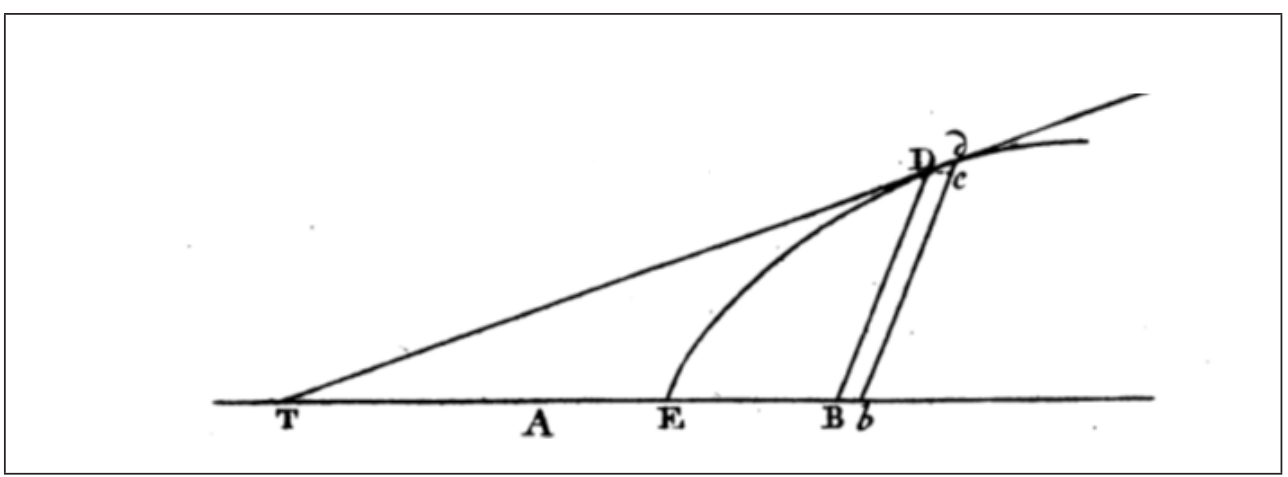

Figure 4: the diagram used by Newton to explain his method of the tangents. From Newton 1746, p. 126.

The problem is to trace the tangent to a given curve in $D$. He considers a reference frame, in which $B D$ is the ordinates' axis and $A B$ the abscissas', (the angle $A B D$ is given, but not necessarily right). Let us suppose that the ordinate moves of an indefinitely small space (spatium indefinite parvum is the Latin expression, Newton 1746, p. 89) $B b$ along the abscissas' line. The momentum of the ordinate will be $c d$, the momentum of the abscissas is $B b$ (or $D c$, parallel to $B b$ ) itself. Now, if one produces $D d$ - which is the line sawing the curve in $D$ and $d$ - till it touches $A B$ in $T$, the triangles $D c d$ and $D B T$ are similar, so that:

$$
T B: B D=D c: c d
$$

The equation of the curve is given, thus the relation between the momenta - and hence between the fluxions - $D c: c d$ is given. It is enough to take $T B$ to $B D$ in the same ratio as $D c$ to $c d$ and the tangent can be traced. This is the general method. Newton supplies several examples.

Let us see how he applies his method to a curve which is difficult to be treated in the courses concerning the functions of one variable, because such a curve is not a function in the modern sense of the term: the conchoid of Nicomedes. Let us remind the readers that the conchoid of Nicomedes is generated like this: let us consider a line $A B$ and a point $G$ out of the line, which are the basis and the pole of the conchoid. Let us establish an interval of length $k$ and trace a straight line $g$ which rotates around the pole. In any position of $g$, it will saw a different point of $A B$. Let us consider, for example the point $L$ and, along $g$, let us trace, from $L$ and in the opposite direction with respect to $L$, the two segments of length $k: L D=L d=k$. The two points identified by the second extremes of the two segments belong to the conchoid, which, hence, has two branches. The form of the conchoid varies according to the quantitative relations among the elements we have introduced, but the considerations concerning the tangent are the same for any form of the conchoid. The line $A B$ is the asymptote of the conchoid. 


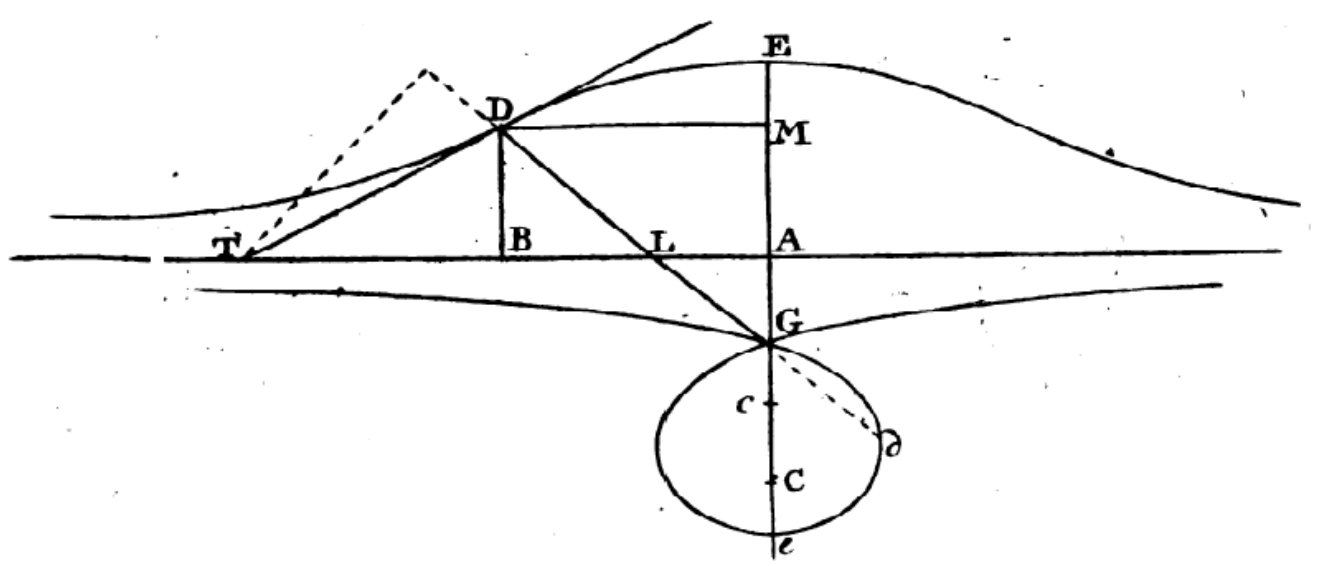

Figure 5: The conchoid: figure used by Newton to describe how to trace the tangent to the curve. From Newton, 1746, p. 126.

Thence, Newton reasons like this: let $G$ be the pole, $A T$ the asymptote, $L D$ the interval. Newton poses $G A=b, L D=c, A B=x ; B D=y$. The point $B$ is hence the origin of the reference frame. The triangles $D B L$ and $D M G$ are similar. Thus, it is

namely

$$
L B: B D=D M: M G
$$

Thus,

$$
\sqrt{c^{2}-y^{2}}: y=x:(b+y)
$$

$$
x y=(b+y) \sqrt{c^{2}-y^{2}}
$$

Now Newton poses $z=\sqrt{c^{2}-y^{2}}$ and the previous equation becomes

with the condition

$$
b z+y z=x y \quad \text { A) }
$$

$$
z^{2}=c^{2}-y^{2} \quad \text { B) }
$$

It is hence possible to write the system of the fluxions-equations of A) and B):

$$
\left\{\begin{array}{l}
b \dot{z}+y \dot{z}+\dot{y} z=\dot{y} x+y \dot{x} \\
\dot{z} z+\dot{y} y=0
\end{array}\right.
$$

If we obtain $z$ from the latter equation of the system and replace its value in the former, we get the equation 
Paolo BUSSOTTI. Differential calculus: The use of Newton's methodus fluxionum et serierum infinitarum in an education context

PROBLEMS

OF EDUCATION

IN THE $21^{\text {st }}$ CENTURY

Volume 65, 2015

$$
-\frac{b \dot{y} y}{z}-\frac{\dot{y} y^{2}}{z}+\dot{y} z=\dot{y} x+\dot{x} y
$$

which is the same as the proportion

$$
y:\left(z-\frac{b y}{z}-\frac{y^{2}}{z}-x\right)=y: \dot{x}
$$

But, if $T$ is the point in which the tangent meets the abscissas, from the theory previously expounded, it is

$$
y: \dot{x}=B D: B T
$$

Since $B D=y$, from C) and D), it follows that

$$
B T=z-x-\frac{b y}{z}-\frac{y^{2}}{z}=z-x+\frac{-y(b+y)}{z}
$$

Furthermore, $x=A B, z=B L, y=B D=M A, b+y=G M$, so that

$$
-\overline{B T}=A L+\frac{B D \cdot G M}{B L}
$$

Finally: all the elements on the right side of this equation are given, so that $B T$ can be constructed, hence the point $T$ can be determined. Joining $T$ with $D$, the tangent to the conchoid is found. The negative sign indicates that the point $T$ is in the opposite position of $A$ in respect to $B$.

Didactical considerations: the ratio between $y$ and $x$ is used by Newton to find the point in which the tangent saws the abscissas' axis. In other terms, such ratio supplies the angular coefficient of the tangent, which is exactly what is commonly taught, namely that the derivative in a given point of a function offers the value of tangent's angular coefficient. Thence, although Newton's differential calculus is based upon a relatively limited number of notions and many concepts were not yet developed, the consideration of $x$ and $y$ as mutually independent variables and the use made by Newton of his notions and procedures is extremely clear, concise and fruitful. In the case of the conchoid, the introduction of the further variable $z$ also allows Newton to avoid the calculation of a fluxion involving a square root, with the difficulties which can arise from an equation in which roots are involved. It is exactly the idea to treat - in a correct mathematical manner - $z$ as an independent variable, though in fact, $z$ is a function of $y$, which enables Newton to reach easily the tangent to the conchoid. Using different approaches, the problem of tracing the tangent to the conchoid is not easy (for ingenious solutions see, i.e., Fermat, 1896, pp. 161-163). Therefore, in a didactical use of Newton's Methodus fluxionum et serierum infinitarum, it is necessary to point out the advantages to consider the variables as mutually independent and the possibility to face, from the beginning of a course of mathematical analysis, a series of problems concerning more than one variables which are normally addressed in the successive courses, when the partial derivatives are introduced. Furthermore, this approach is intuitive and can induce the students to actively solve similar problems, for which a basic technique is offered, but numerous personal procedures (as the introduction of the variable $z$ ) can he invented to simplify such basic technique. Thence, this can favour the creativity of the pupils/students. Obviously, the limits of such approach must not be hidden, but this is a supplementary way of thinking and approaching the mathematical problems, which can be useful in the mathematical background of the pupils/students. 
Newton continues this part of his work dealing with the problems of the points of flexures in a curve and proposes some interesting considerations concerning the links between the tangents-problem and the areas-problem (he will develop this question in successive sections of his work).

\section{The Curvature of a Line in a Given Point}

The concept of curvature in a point is, in general, studied in the second course of mathematical analysis at the university. Only to remind the reader some basic notions. Given a curve in a plane $f(x, y)=0$, indicated by $d s=\sqrt{d x^{2}+d y^{2}}$ the arc-differential and with $\varphi$ the angle, at a given point $P$ of the curve, between the tangent-versor $\mathbf{t}$ and the $x$-axis, the curvature in the point $P$ is given by

$$
c=\left|\frac{d \varphi}{d s}\right|
$$

It is easy to transform this formula into

$$
c=\frac{\left|d^{2} x / d y^{2}\right|}{\left[1+(d x / d y)^{2}\right]^{3 / 2}}
$$

If the curve is expressed by means of a parametric equation in function of a variable $t$, it is

$$
c=\frac{|\dot{x} y-y x|}{\left[\begin{array}{lr}
.2 & .2 \\
x+y
\end{array}\right]^{3 / 2}}
$$

The curvature- or osculating circle is considered the circle which best approximates a curve in a given point and whose radius is the reciprocal of the curvature. The osculating circle is a second order approximation of a curve, whereas the tangent is a first order approximation, thence the approximation given by the osculating circle is more precise. Several considerations drawn from the vectorial analysis could be added and, obviously, the concept of curvature can be presented inside the general picture of the differential geometry. For my aims, these brief references are enough.

Newton, before dealing with analytical considerations, offers the geometrical terms of the problem. 
PROBLEMS

OF EDUCATION

IN THE $21^{\text {st }}$ CENTURY

Volume 65,2015

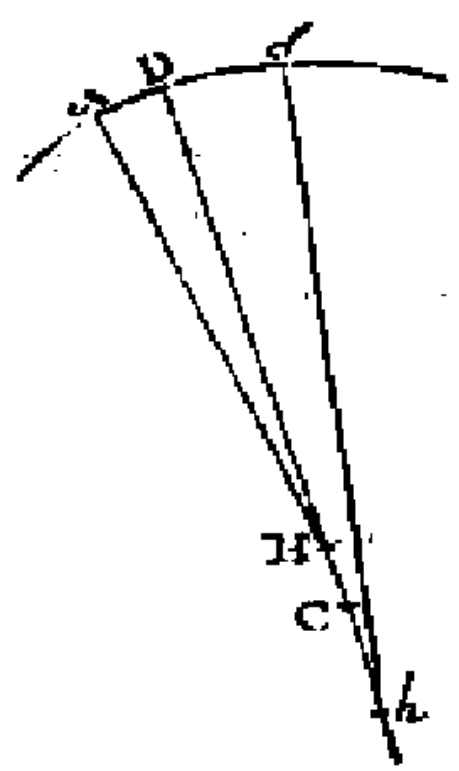

Figure 6: The figure used by Newton to introduce his considerations on the curvature of a line in a given point $\boldsymbol{D}$. From Newton 1736, p. 60.

Let us consider three point $\delta, \mathrm{D}, \mathrm{d}$ of the given curve. Since it is possible to trace the tangents to the curve in these points, it is also possible to trace the perpendiculars to the curve. Let the perpendicular from $\delta$ and $D$ meet in $H$ and from $\delta$ and $d$ in $h$. Newton prosecutes: when the three perpendiculars meet, the three points $\delta, \mathrm{D}, \mathrm{d}$ coincide with $D$. Let us suppose this happens in the point $C$, the segment $C D$ is the radius of the curvature circle in $D$ and $C$ is its centre. Given this construction, Newton specifies that no other circle can be inserted, in the point $D$, between the curve and the osculating circle. He adds there are several procedures to trace the curvature circle, but the easiest and methodical consists in resorting to the fluxions-calculus like this:

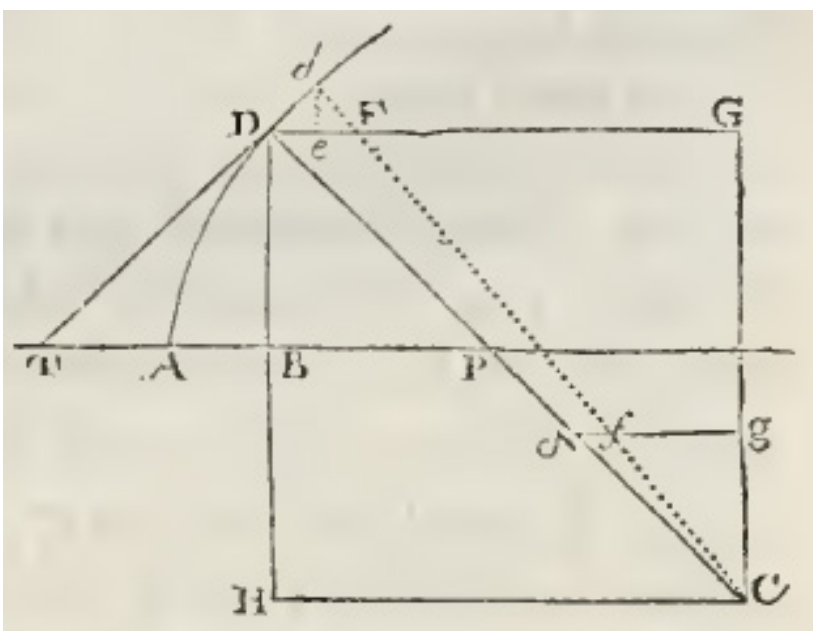

Figure 7: The method used by Newton to determine the radius of the curvaturecircle. From Newton 1736, p. 61. 
The positions are as follows:

$A D=$ curve, $D=$ point in which we calculate the curvature; $B=$ origin of the orthogonal reference frame; $A B=x$-axis; $B D=y$-axis; $D T=$ tangent to the curve in $D$ ( $T$ belongs to the abscissas' axis); $D C=$ perpendicular to the curve; $C=$ the centre of curvature supposed known (it is typical of the analytical method to suppose an unknown quantity is known and, after some steps, to show it can be, in fact constructed with the available data); $D G, H C$ parallel and $H D$, $C G$ perpendicular to the abscissas.

Explanation: let a segment of any length $C g$ be considered in $C G$ and let $g \delta$ be perpendicular to $C g$. It is

$$
C g: g \delta=T B: B D
$$

But, because of Newton's theory of the tangents, it is

$$
T B: B D=\dot{x}: \dot{y}
$$

Let us now suppose that the point $D$ moves along the curve by an infinitely small distance (infinite parum progredi is the original Latin expression, Newton 1746, p. 107) reaching $d$. Let $d e$ be perpendicular to $D G$ and $C d$ perpendicular to the curve. Let $C d$ meet $g \delta$ in $f$ and $D G$ in $F$. Therefore: $D e=$ momentum of the abscissas; $d e=$ momentum of the ordinates; $\delta \mathrm{f}=$ momentum of $g \delta$.

From the right triangle $D d F$, it follows

$$
D F=D e+\frac{d e^{2}}{D e}
$$

(for, applying Euclid's theorems, it is $d e^{2}=D e \cdot e F$, but $e F=D F-D e$ ).

Once determined $D F$ as a function of the momenta and, hence, of the fluxions of $x$ and $y$, the point $C$ can be determined. For, let us pose $C g=1$ (this does not compromise the generality of the procedure) and $g \delta=z$. It is

$$
C g: g \delta=D e: d e
$$

because the triangles $D d e$ and $C g \delta$ are similar. Thence

$$
1: z=\dot{x}: \dot{y}
$$

So that $z=\frac{y}{\dot{x}}$.

From 1) it follows that

$$
D F=o \dot{x}+\frac{o y}{\dot{x}}
$$


Paolo BUSSOTTI. Differential calculus: The use of Newton's methodus fluxionum et serierum infinitarum in an education context

PROBLEMS

OF EDUCATION

IN THE $21^{\text {st }}$ CENTURY

Volume 65,2015

In conclusion:

$$
C g: C G=\delta f: D F=o z:\left(o x+\frac{.2}{.}\right)
$$

which means

$$
C G=\frac{.2 .2}{x+y}
$$

as $C g=1$. This allows us to determine the point $C$.

By means of easy passages Newton determines the length of the curvature radius $D C$ and he is able to express it as a function of $z$ and $z$. To be precise, once posed $x=1$ :

$$
D C=\frac{\left(1+z^{2}\right)^{\frac{3}{2}}}{z} .
$$

Didactical considerations: first of all a technical question. Let us take into account that

$z=\frac{y}{}$. Replacing this value in the expression for $C G$, without posing $\dot{x}=1$, one gets $x$

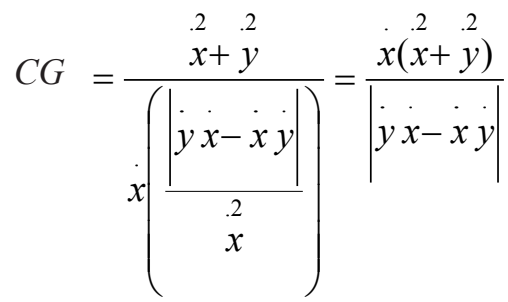

Since

it is

$$
C G: D G=\dot{x}: \dot{y}
$$

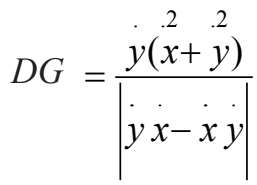


and applying Pythagoras' theorem

which is exactly our expression for the curvature radius.

A particular consideration concerns the use of geometry inside mathematical analysis. Newton arrived at determining the curvature-radius also by means of elementary geometrical reasoning concerning the similarities of the triangles. Nowadays the tendency is to present every aspect of the theory through analytical argumentations without taking into account the geometrical aspect. Whereas, the use of geometry is a precious help to provide an intuitive support to the analytical procedures. For example, in Newton's reasoning the link between the tangent to a curve in a given point and the osculating circle in that same point is well expressed. The resort to geometry might help the pupils/students to catch the meaning of the operations they are learning. A critical interest could be developed in understanding the methods by Newton, because he applies the similarity between finite and infinitesimal triangles, too. This aspect ought to attract the attention of the pupils/students since, beyond the use of geometry, they could ask what kind of mathematical entities the infinitesimal triangles, with which Newton deals, are. Is this expressed by Newton a potential conception of infinity? Is it an actual conception? Or is this another kind of idea? This doubt could be originated by the fact, that Newton seems to consider infinitesimal triangles as Ded or DFd in the last figure, as given entities. It should be explained that Newton did not conceive these infinitesimals as actual quantities, but that they are as a fiction: in a certain phase of his reasoning Newton needs to consider these triangles as given, but he does not think they have an existence as a finite triangle. They are a fiction useful to develop his reasoning. This use Newton makes of the infinitesimals is a quite significant and instructive example of how some entities can be used in mathematics: they do no exist in the same sense in which other mathematical entities exist, but they are "imaginary" objects useful to carry out an argumentation. This can help the pupils/ students to enter into the logic and the methodology of mathematics.

Other interesting questions faced by Newton in his Methodus fluxionum - i.e: points of flexures, integration by series, solution of differential equations, calculation of areas and lengths of the curves - could be dealt with, but, due to the lack of room, I limit to the already analysed questions on the differential calculus, without addressing the integral calculus.

\section{Conclusion: Teaching Itineraries Based on Newton's Work}

The expounded material, drawn from Newton's Methodus fluxionum et serierum infinitarum can be taught in four-five lessons following two different possible teaching-itineraries, according to the preferences of the teacher/professor.

First itinerary (more theoretically oriented).

Lesson 1: a) introduction of the concept of fluxion and fluent; b) discussion on the kind of infinitesimals used by Newton (actual, potential, or "fictions"?); c) rules of fluxions-calculus.

Lesson 2: application of the fluxions-calculus to the problems of maxima-minima and tangents.

Lesson 3: introduction of the concept of curvature centre and osculating circle in connection to the fluxions-calculus.

Lesson 4: the development of mathematical expressions in series of powers as an introduction to the problems of Taylor series and to the integration by series.

Lesson 5: critical considerations on the great potential (in particular the fact that many problems concerning one, two or more variables can be treated in a uniform way), but also 
PROBLEMS

OF EDUCATION

IN THE $21^{\text {st }}$ CENTURY Volume 65,2015

64

on the theoretical limits, of the introduced methods. Necessity of a more abstract, formal and general approach.

Second itinerary (more problem-solving oriented).

Lesson 1: the teacher/professor enters into the classroom, draws a curved line on the blackboard and asks the pupils/students how they think to trace a tangent from a point of the curve. Here a discussion with the class can be born. The teacher/professor should show the similarities between the tangent and the maxima-minima problems.

Lesson 2: introduction of the concept of fluxion, specifically related to the problems addressed in the first lesson.

Lesson 3: extension of the problems treatable by the fluxions-calculus. Reference the question of the osculating circle.

Lesson 4 and lesson 5: the teacher/professor poses to the pupils/students the problem to write an algebraic equation $f(x, y)=0$ expressing a variable as a series of powers of the other variable. The answer to this question might open a discussion concerning the general possibility to separate the variables by following Newton's method of infinite series (hence, also including some kinds of differential equations), as well as an introduction to the integration by series.

Lesson 6: as the lesson 5 of the first itinerary.

These two concrete itineraries (and numerous others could be conceived) prove history of mathematics - or better an analysis of the way in which e certain problem has been faced by the great mathematicians in the past - is a fundamental support for the pupils/students to enter in contact with a more intuitive, problems-based and - in many cases - geometrical way of thinking. This might help them to catch the real meaning of some mathematical methods and procedures, the way and the reasons why such procedures and theories are born. Obviously: after this phase, the formalization in modern terms is necessary to get a precise and perspicuous view on the problems. In this way, the pupils/students are driven inside a fascinating mathematical world, full of interesting problems and of ingenious solutions. After that, the formal approach will be appreciated, too. It will be perceived as something necessary and not as a sort of arbitrary imposition. The example of maxima, minima, tangent is particularly significant, but other initial subjects, on which to base the first steps of a course of mathematical analysis, can be chosen.

A possible objection is that there is no sufficient time to develop such approach to the teaching of mathematics. The answer is easy: especially in the context of the high school, it is not necessary to develop a program in which many subjects are dealt with. Rather, it is important that these subjects are faced in a profound and comprehensible manner. The quality of the instruction is more significant than the quantity of the imparted notions. In this sense, history of mathematics can be a fundamental support.

Finally, the specific meaning of using Newton's Methodus fluxionum et serierum infinitarum can be summarized like this:

1) Development in series of expressions and equations: this is an instructive introduction to the theory of series and of integrals' calculation by series. The extreme efficacy of this method should be pointed out as well as its limitation. In particular: a) a theory of the integration in a proper sense is not developed, which, historically, will mark the superiority of the "continental" mathematical analysis in respect to the analysis developed in England during the $18^{\text {th }}$ and the beginning of the $19^{\text {th }}$ century; b) the problem of the convergence of the series was, in fact, not faced by Newton.

2) With regard to the fluxions: comparison between fluxions, Leibniz's differentials and our derivatives. This is an important educative step. It is useful to understand that many mathematical results have been reached without a very theory of functions and without an explicit resort to the concept of limit. On the other hand, Newton had two brilliant ideas: to treat the variables as a function of the time, to treat them as mutually independent. This allowed 
him a prolific and easy approach to several problems. What are the relations between such an approach and the modern-formal one?

3) With regard to the concept of infinity: the limit in modern analysis is clearly based on a potential conception of infinity. What kind of infinity are Newton's evanescent triangles? This discussion could be intriguing for the pupils/students.

4) The use of geometry simplifies many procedures and makes them clearer and more intuitive. I am convinced that geometrical considerations have to be reintroduced in an educative context. Newton's approach is paradigmatic in this sense.

\section{Acknowledgement}

The author acknowledges Professor Dr. Niccolò Guicciardini and the anonymous reviewer for their precious indications.

\section{References}

Bussotti, P. (2014). The possible relations between history of mathematics and mathematics education. Science and Technology Education for the 21 $1^{\text {st }}$ Century. Research and Research Oriented Studies. Proceedings of the $9^{\text {th }}$ IOSTE Symposium for Central and Eastern Europe. Hradec Králové: Gaudeamus, 29-41.

Fermat, P. (1896). Maxima et minima. In P. Fermat, Oeuvres de Fermat, Tome I (pp. 133-179). Pairs: Gauthier-Villars. Retrieved from ftp://ftp.bnf.fr/621/N6213144_PDF_1_-1DM.pdf.

Leibniz, G. W. (1684, 1962). Nova Methodus pro Maximis et Minimis, itemque Tangentibus, quae nec fractis nec irrationales quantitates moratur, et singulare pro illis calculi genus. In G. W. Leibniz, Mathematische Schriften (pp. 220-226). Hildesheim: Georg Holms.

Newton, I. (1671, 1736). The method of fluxions and infinite series. London: Henry Woodfall. Retrieved from https://archive.org/details/methodoffluxions00newt.

Newton, I. (1726, 1739-1742). Philosophiae naturalis principia mathematica, auctore Isaaco Newtono, Eq. Aurato. Perpetuis commentariis illustrate, communi studio pp. Thomae le Seur et Francisci Jacquier ex Gallicana Minimorum Familia, matheseos professorum. Geneva: Barillot et filii.

Newton, I. (1746). Methodus fluxionum et serierum infinitarum, in I. Newton, Opuscula mathematica, I (pp. 29-200). Lausanne et Genevae: Marcum Michaelem Bousquet. Retrieved from https://archive.org/details/OpusculaMathematica.

Newton, I. (1701, 1746). Tractatus de quadratura curvarum. In I. Newton, Opuscula mathematica, I (pp. 201-245). Lausanne et Genevae: Marcum Michaelem Bousquet. Retrieved from https://archive. org/details/OpusculaMathematica.

Suzuki, J. (2005). The Lost Calculus (1637-1670): Tangency and Optimization without Limits. Mathematics Magazine, 78(5), 339-353. Retrieved from http://www.maa.org/programs/maa-awards/ writing-awards/the-lost-calculus-1637-1670-tangency-and-optimization-without-limits.

\section{Advised by Naglis Švickus, SMC “Scientia Educologica”, Lithuania}

Received: May 15, 2015

Accepted: June 18, 2015

Paolo Bussotti
Ph.D., Contract Professor for History of Science, University of Udine, via Palladio 8, 33100 Udine, Italy. E-mail: paolobussotti66@gmail.com Website: http://www.scienzainrete.it/documenti/autori/paolo-bussotti
OF EDUCATION
IN THE 21 $1^{\text {st }}$ CENTURY
Volume 65, 2015 